\title{
DDP-MECY Regimen
}

National Cancer Institute

\section{Source}

National Cancer Institute. DDP-MECY Regimen. NCI Thesaurus. Code C162004.

A chemotherapy regimen consisting of cisplatin followed by high-dose methotrexate with leucovorin rescue, and cyclophosphamide that may be used in the treatment of ovarian cancer. 